\title{
Revisiting Decomposition Analysis of Geometric Constraint Graphs
}

\author{
R. Joan-Arinyo, A. Soto-Riera, S. Vila-Marta, J. Vilaplana-Pastó \\ Universitat Politècnica de Catalunya \\ Departament de Llenguatges i Sistemes Informàtics \\ Av. Diagonal 647, 8a, E-08028 Barcelona \\ e-mail: [robert, tonis, sebas, josep]@lsi.upc.es
}

\begin{abstract}
Geometric problems defined by constraints can be represented by geometric constraint graphs whose nodes are geometric elements and whose arcs represent geometric constraints. Reduction and decomposition are techniques commonly used to analyze geometric constraint graphs in geometric constraint solving.

In this paper we first introduce the concept of deficit of a constraint graph. Then we give a new formalization of the decomposition algorithm due to Owen. This new formalization is based on preserving the deficit rather than on computing triconnected components of the graph and is simpler. Finally we apply tree decompositions to prove that the class of problems solved by the formalizations studied here and other formalizations reported in the literature is the same.
\end{abstract}

Keywords Constraint solving, geometric constraints, graph-based constraint solving.

\section{Introduction}

Geometric problems defined by constraints can be represented by geometric constraint graphs whose nodes are geometric elements and whose arcs represent geometric constraints.

For application with potentially large constraints systems, the efficiency of the algorithms for solving the system at hand is an important issue. To decide on the suitability of a given constraint solving method, its correctness must be proved and the class of problems the method can solve should be characterized.

Many attempts to provide general, powerful and efficient methods for solving systems of geometric constraints have been reported in the literature. For an extensive review in geometric constraint solving refer to Fudos [6] and Durand [3].

Among the existing methods we focus on two techniques commonly used to analyze geometric constraint graphs in geometric constraint solving, generically known as decomposition and reduction, respectively. More specifically we are interested in decomposition and reduction where the analysis is based on a direct geometric interpretation. There are other approaches. See for example Hoffmann et al. [7] for a flow-based decomposition algorithm.

In [13], Owen described a top-down algorithm for computing a decomposition of an arbitrary graph. The algorithm recursively splits the graph into split components, [8]. The algorithm terminates when the graphs cannot be split further. At the end of the analysis the original graph has been decomposed into a set of triangles.

Fudos and Hoffmann, [6], reported on a graph-constructive approach to solving systems of geometric constraints. The method is based on an analysis of the constraint graph that derives a sequence of construction steps that sequentially places the geometric elements in the problem with respect to each other. The analysis has two parts. The first part is a bottom-up reduction analysis where each step in the sequence corresponds to positioning three rigid geometric bodies that pairwise share a geometric element, point or line. The second part is a top-down decomposition analysis that produces a sequence of decompositions that correspond to a reverse sequence of rigid geometric bodies.

In this paper we reformulate the algorithm reported by Owen in [13] to solving geometric constraint problems based on the decomposition analysis of the constraint graph. First we introduce the concept of deficit associated with a constraint graph. The deficit measures the distance between a given constraint graph and a wellconstrained graph induced by the same set of nodes. The deficit allows us to avoid the need for computing triconnected components yields a simpler algorithm both conceptually and from a computational runtime point of view. Then we recall the tree decomposition of a constraint graph, a tool that has is useful to conceptually analyze constraint graphs. Finally tree decompositions are applied to characterize the class of problems solved by the decomposition analysis studied here and to prove that different formalizations solve the same class of problems.

Section 2 reviews basic concepts from graph theory and geometric constraint graphs. Section 3 deals with decomposition analysis. First we recall Owen's algorithm, then we present the new formalization of the algorithm. Section 4 presents the tree decomposition of a constraint graph. Section 5 is devoted to characterize the class of problems solved by the decomposition analysis studied here and discusses the equivalence of different formalizations. We close with a brief summary in Section 6.

\section{Preliminaries}

In this section we recall basic terminology of graph theory, the concept of geometric constraint graph associated to a geometric problem defined by constraints, and some definitions related to geometric constraint graphs.

\subsection{Graph Concepts}

First we recall some basic terminology of graph theory that will be used in the rest of the paper. For an extensive treatment see [2] and [8].

A graph $G=(V, E)$ is said to be connected if every vertex is connected to every other node by at least one path of edges. We say that a node $a$ of a connected graph $G$ is an articulation node if by removing $a$, the graph splits into two or more disconnected subgraphs. If $a$ is an articulation node in $G$, then there are two vertices 
one of the virtual edges introduced in the first step. Note that virtual edges are always incident to separation pairs.

Then the first step is recursively applied to the transformed split components. The algorithm terminates when the graphs cannot be split further. If any triconnected graph with more than three vertices remains, the problem cannot be solved quadratically.

At the end of the analysis, the original graph has been decomposed into a set of triangles whose edges are either original edges or virtual edges.

If function SplitComponents (G) computes the split components of $G$, function Reducible (g) checks whether a split component should be further subdivided, and function Reduce (g) removes unneeded virtual edges of graph $g$, Owen's algorithm can be written as shown in Figure 2.

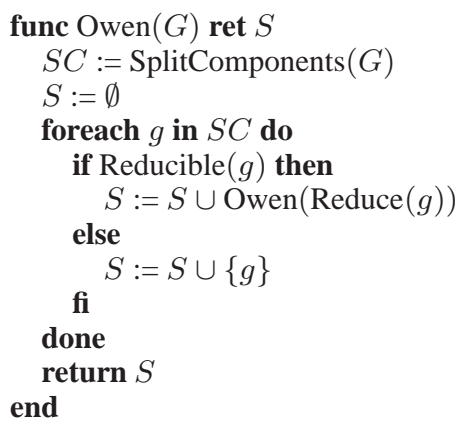

Figure 2: Owen's analysis algorithm.

The analysis process followed by Owen's analysis algorithm is illustrated in Figure 3. Virtual edges are shown in dashed lines.

\subsection{The New Formalization}

To decompose a graph, Owen's method uses the algorithm for finding triconnected components reported by Hopcroft and Tarjan in [8]. which is based on preserving graph connectivity. As a result, the split components generated by the decomposition include extra virtual edges. To recursively apply the decomposition process, Owen's algorithm must remove these extra virtual edges.

In what follows we will present an algorithm to decompose a constraint graph in triconnected graphs with exactly three vertices, that is, triangles. The algorithm is based on a divide and conquer strategy which preserves the constraint graph property of being wellconstrained. The resulting algorithm is conceptually simple and easy to implement.

As in [13] and [6], the algorithm will be based on subdividing the constraint graph into two separating graphs induced by a separation pair. With the aim of clearly stating a subdivision criterion, we start by giving some definitions and deriving properties which relate wellconstrained graphs with their separating graphs.

Definition 3.1 Let $G=(V, E)$ be a geometric constraint graph. We define the Deficit function associated with $G$ by

$$
\operatorname{Deficit}(G)=(2|V|-3)-|E|
$$

The function Deficit computes the difference between the number of edges needed for a constraint graph to be wellconstrained and its actual number of edges. Note that if $G$ is not overconstrained, $\operatorname{Deficit}(G) \geq 0$
Lemma 3.2 Let $G$ be a constraint graph and $G^{\prime}$ and $G^{\prime \prime}$ separating graphs. Then

$$
\operatorname{Deficit}(G)=\operatorname{Deficit}\left(G^{\prime}\right)+\operatorname{Deficit}\left(G^{\prime \prime}\right)-1
$$

Proof

By definition, Deficit $(G)=(2|V|-3)-|E|)$. Since $G^{\prime}$ and $G^{\prime \prime}$ are separation graphs of $G$, then $|V|=\left|V^{\prime}\right|+\left|V^{\prime \prime}\right|-2$ and $|E|=\left|E^{\prime}\right|+\left|E^{\prime \prime}\right|$. Therefore,

$$
\begin{aligned}
\operatorname{Deficit}(G) & =2\left(\left|V^{\prime}\right|+\left|V^{\prime \prime}\right|-2\right)-3-\left(\left|E^{\prime}\right|+\left|E^{\prime \prime}\right|\right) \\
& =\left(2\left|V^{\prime}\right|-3-\left|E^{\prime}\right|\right)+\left(2\left|V^{\prime \prime}\right|-3-\left|E^{\prime \prime}\right|\right)-1 \\
& =\operatorname{Deficit}\left(G^{\prime}\right)+\operatorname{Deficit}\left(G^{\prime}\right)-1
\end{aligned}
$$

Lemma 3.3 Let $G$ be a wellconstrained graph and $G^{\prime}$ and $G^{\prime \prime}$ separating graphs. Then if Deficit $\left(G^{\prime}\right)>\operatorname{Deficit}\left(G^{\prime \prime}\right), G^{\prime}$ is underconstrained and $G^{\prime \prime}$ is wellconstrained.

\section{Proof}

Since $G$ is wellconstrained, $\operatorname{Deficit}(G)=0$ and separation graphs, $G^{\prime}$ and $G^{\prime \prime}$, are not overconstrained, that is, Deficit $\left(G^{\prime}\right) \geq 0$ and $\operatorname{Deficit}\left(G^{\prime \prime}\right) \geq 0$. From Lemma 3.2 Deficit $(G)=\operatorname{Deficit}\left(G^{\prime}\right)+$ $\operatorname{Deficit}\left(G^{\prime \prime}\right)=1$. Thus Deficit $\left(G^{\prime}\right)+\operatorname{Deficit}\left(G^{\prime \prime}\right)=1$. Then, either $\operatorname{Deficit}\left(G^{\prime}\right)=1$ and $\operatorname{Deficit}\left(G^{\prime \prime}\right)=0$, which means that $G^{\prime}$ is underconstrained and $G^{\prime \prime}$ wellconstrained or vice versa.

Definition 3.4 Let $G$ be a wellconstrained constraint graph and $G^{\prime}$ and $G^{\prime \prime}$ separating graphs. The modified split graphs, $G_{1}$ and $G_{2}$, of $G$ are defined as follows. If Deficit $\left(G^{\prime}\right)>\operatorname{Deficit}\left(G^{\prime \prime}\right)$ then

$$
G_{1}=\left(V\left(E^{\prime}\right), E^{\prime} \cup\{(a, b)\}\right) \quad \text { and } \quad G_{2}=G^{\prime \prime}
$$

otherwise

$$
G_{1}=G^{\prime} \quad \text { and } \quad G_{2}=\left(V\left(E^{\prime \prime}\right), E^{\prime \prime} \cup\{(a, b)\}\right)
$$

Lemma 3.5 Let $G=(V, E)$ be a constraint graph and, $G_{1}=$ $\left(V_{1}, E_{1}\right)$ and $G_{2}=\left(V_{2}, E_{2}\right)$ be modified split graphs. Then $\operatorname{Deficit}(G)=\operatorname{Deficit}\left(G_{1}\right)+\operatorname{Deficit}\left(G_{2}\right)$.

\section{Proof}

Now $|E|=\left|E_{1}\right|+\left|E_{2}\right|-1$. Apply proof of Lemma 3.2.

Definition 3.6 An s-tree is a binary tree such that:

1. the root is a constraint graph $G$,

2. for each node in $G$ the root of their sons are the modified split graphs $S_{1}$ and $S_{2}$ of $G$, and

3. the leaves are either triangles or triconnected graphs.

Let Triconnected $(G)$ be a function that tests whether a graph has a separation pair, SeparatingGraphs $(G)$ a function that computes the separating graphs of $G$, (Recall that separating graphs do not include virtual edges), and AddVirtualEdge $(G)$ a function that adds a virtual edge incident to the separation pair used to compute the split graph $G$. Then the decomposition analysis algorithm based on preserving deficits of graphs can be written as shown in Figure 4.

The input to the algorithm is a graph $G$ associated to a geometric constraint problem. The output is a s-tree $S$ whose root is $G$. 


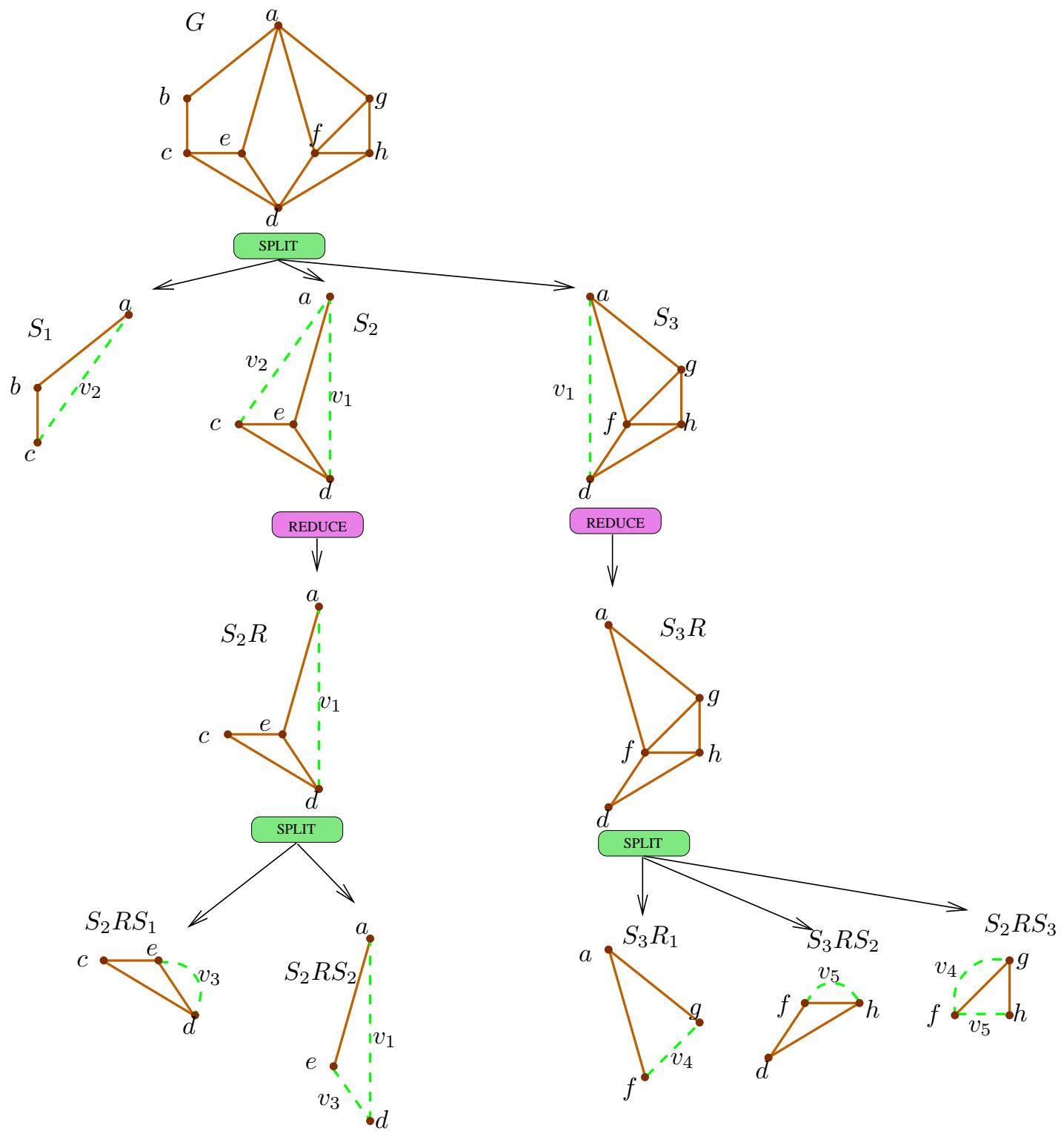

Figure 3: Owen's algorithm computation applied to an example graph. 


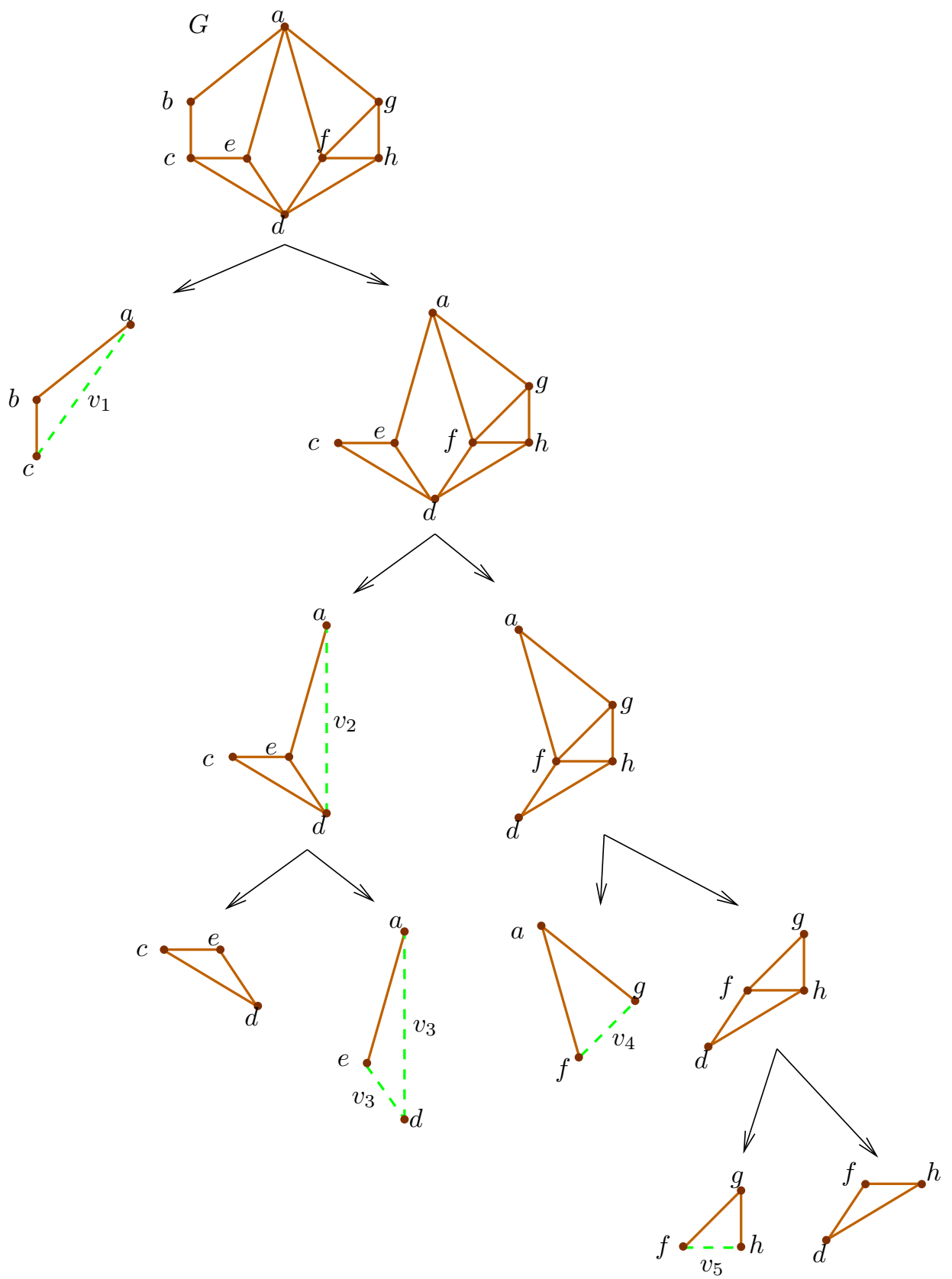

Figure 5: Decomposition analysis generated by the new algorithm on the example graph in Figue 3. 


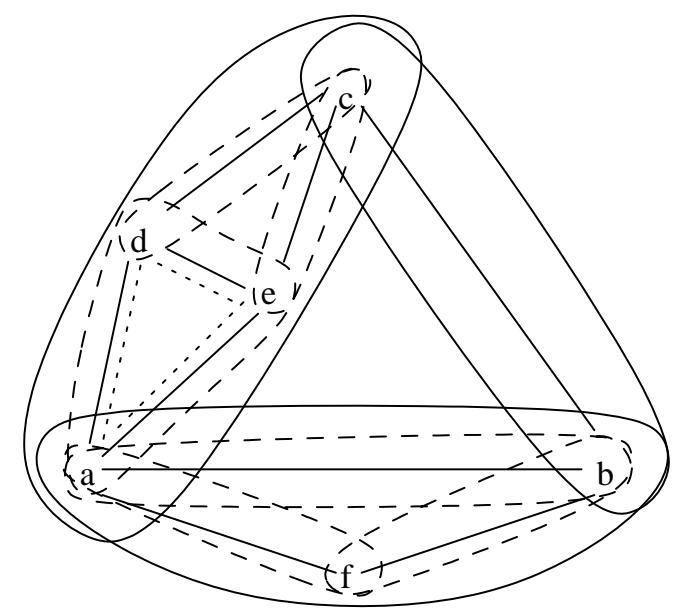

Figure 11: Collection of set decompositions of the graph in Figure 9.

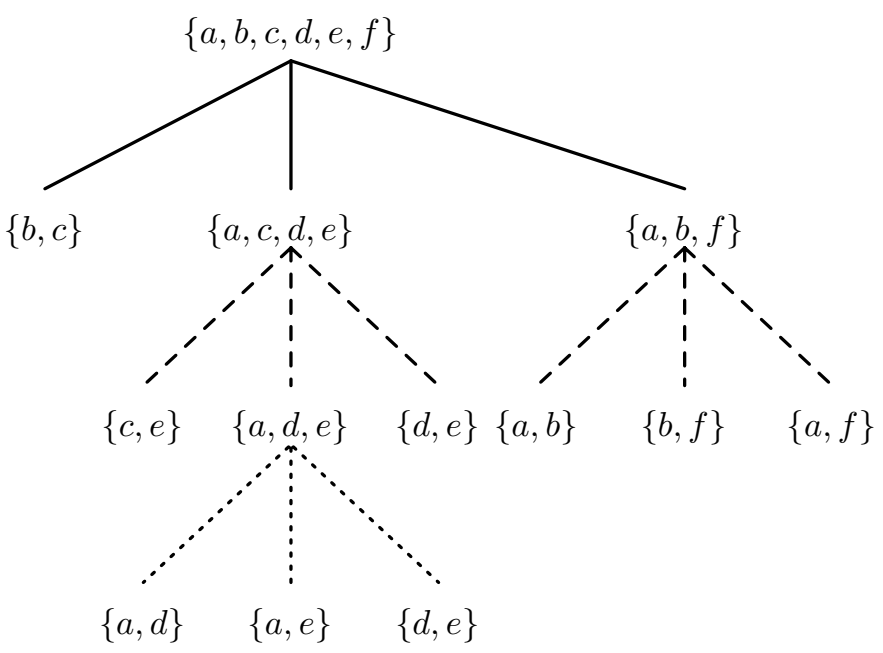

Figure 12: Tree decomposition of the graph in Figure 11. the leaves are triangles or triconnected graphs with no articulation pairs.

Definition 5.1 We say that a constraint graph $G$ is s-tree decomposable if there is a s-tree such that its root is $G$ and all its leaves are triangles.

According to the number of virtual edges in the triangles in the leaf nodes of an s-tree, we classify them in four different types. See the second column in Table 1.

\subsection{Domain Characterization}

To characterize the decomposition analysis studied in Section 3 we prove two lemmas.

Lemma 5.2 If a graph $G$ is tree decomposable, then $G$ is s-tree decomposable.

Proof

Assume that $T$ is a tree decomposition of $G$. We shall proceed by induction on the structure of $T$. Refer to Table 1 .

Induction base: Let $G=(V, E)$ be a graph such that $V=\{a, b, c\}$ and $E=\{(a, b),(a, c),(b, c)\}$. The tree $T$ in the third column of Table 1 is a tree decomposition of $G$. Then the tree in the fourth column is a s-tree whose root is a graph $G=G_{0}$ with just one node representing the triangle $\{a, b, c\}$.

Induction hypothesis: Let $G^{\prime}$ be a subgraph of $G$. If $G^{\prime}$ is tree decomposable then $G^{\prime}$ is s-tree decomposable.

Induction step: If $\left\{C_{1}, C_{2}, C_{3}\right\}$ is a set decomposition of $C$ and $\{a, b, c\}$ the active elements, we have that $C^{\prime}=C-\{a, b, c\}$, $C_{1}^{\prime}=C_{1}-\{a, b\}, C_{2}^{\prime}=C_{2}-\{a, c\}$ and $C_{3}^{\prime}=C_{3}-\{b, c\}$.

Let $G$ be a graph and $T$ a tree decomposition of $G$ such that its root is $\{a, b, c\} \cup C^{\prime}$ and the roots of its subtrees are $\{a, b\} \cup C_{1}^{\prime}$, $\{a, c\} \cup C_{2}^{\prime}$ and $\{b, c\} \cup C_{3}^{\prime}$.

Assume that $C_{1}^{\prime} \neq \emptyset$ and $C_{2}^{\prime}=C_{3}^{\prime}=\emptyset$. Let $G_{1}^{\prime}$ be the subgraph induce by $\{a, b\} \cup C_{1}^{\prime}$ in $G$. Let $T_{1}$ be the tree decomposition of $G_{1}^{\prime}$.

By Lemma 4.3, $(a, b)$ is a separation pair of $G$ thus $G_{1}^{\prime}$ is a separation graph of $G$. Build the other separation graph $G_{2}$ as the graph $G_{2}=\left(V_{2}, E_{2}\right)$ with $V_{2}=\{a, b, c\}$ and $E_{2}=\{(a, c),(b, c)\}$. By Definition 2.1, $G_{2}$ is underconstrained, thus by Lemma 3.3, $G_{1}^{\prime}$ is wellconstrained.

Now build the modified split graphs of $G$ as $G_{0}=\left(V_{2}, E_{2} \cup\right.$ $\{(a, b)\})$ and $G_{1}^{\prime}$.

Since $G_{1}^{\prime}$ is tree decomposable, by the induction hypothesis it is s-tree decomposable. Therefore there is a s-tree, say $S_{1}^{\prime}$, whose root is $G_{1}^{\prime}$. Hence the binary tree whose root is $G$ and whose subtrees are $G_{0}$ and $S_{1}^{\prime}$ is a s-tree. Therefore $G$ is s-tree decomposable.

Applying the same procedure for cases $C_{2}^{\prime} \neq \emptyset$ and $C_{3}^{\prime} \neq \emptyset$ completes the proof.

If function ComputeTriangle $(T)$ computes the triangle associated with a node of a tree decomposition, and function MergeGraphs $\left(G_{1}, G_{2}\right)$ rebuilds a graph from its modified split graphs, Figure 13 shows an algorithm that, based on Lemma 5.2, computes a s-tree $S$ from a tree decomposition $T$ of a graph $G$.

Lemma 5.3 If a graph $G$ is s-tree decomposable, then $G$ is tree decomposable. 


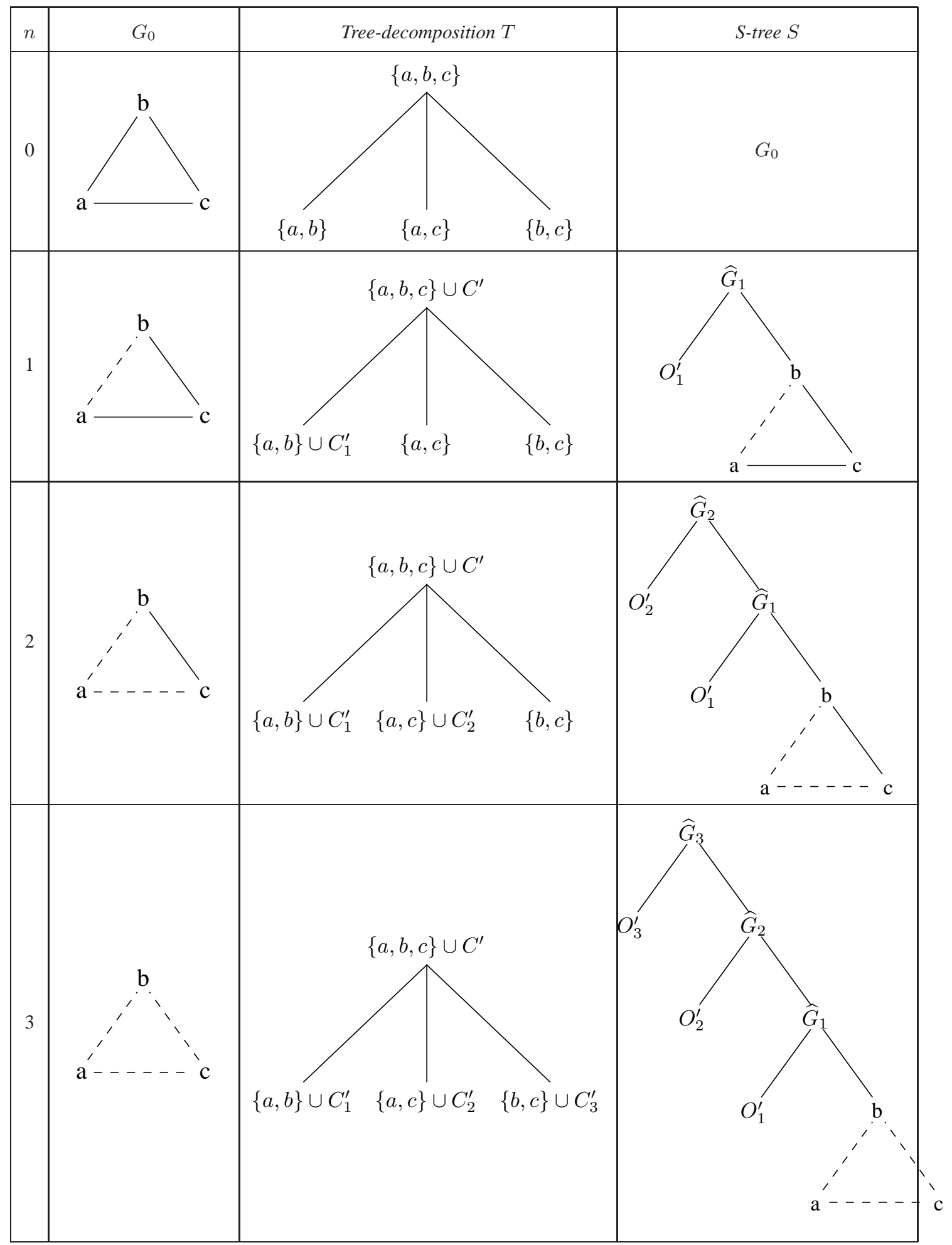

Table 1: Types of interior nodes in a tree decomposition and the equivalent s-tree decomposition. 
func FromTreeToS-Tree $(T)$ ret $S$

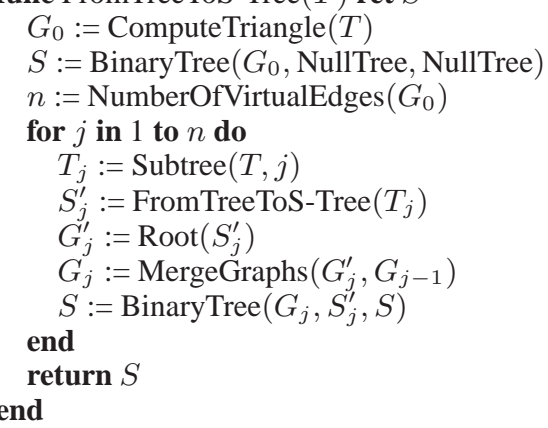

Figure 13: Computing a s-tree $S$ from a tree decomposition $T$.

\section{Proof}

Assume that $S$ is a s-tree whose root is $G$. Again we shall proceed by induction on the structure of $S$. Refer to Table 1 .

Induction base: Let $G=(V, E)$ be a graph such that $V=\{a, b, c\}$ and $E=\{(a, b),(a, c),(b, c)\}$. The s-tree $S$ of $G$ is that given in the fourth column of Table 1 . Then the tree given in the third column is a tree decomposition of $G$.

Induction hypothesis: Let $G^{\prime}$ be a subgraph of $G$. If $G^{\prime}$ is s-tree decomposable then $G^{\prime}$ is tree decomposable.

Induction step: Let $S$ be a s-tree whose root is $G$ and whose subtrees are $G_{0}$ and $S_{1}^{\prime}$.

Assume that $G_{0}=\left(V_{0}, E_{0}\right)$ with $V_{0}=\{a, b, c\}$ and $E_{0}=$ $\{(a, c),(b, c)\} \cup\{(a, b)\}$ and let $G_{1}^{\prime}=\left(V_{1}^{\prime}, E_{1}^{\prime}\right)$ be the root of the s-tree $S_{1}^{\prime}$. By Definition 3.6, $G_{0}$ and $G_{1}^{\prime}$ are the modified split graphs of $G$ with respect to the separation pair $\{a, b\}$. Since $G_{1}^{\prime}$ is stree decomposable, by induction hypothesis it is tree decomposable. Therefore there is a tree decomposition $T_{1}^{\prime}$ of $G_{1}^{\prime}$.

Build a tree decomposition $T$ such that its subtrees are $T_{1}^{\prime},\{a, c\}$ and $\{b, c\}$. See Table 1. Subtrees $\{a, c\}$ and $\{b, c\}$ share the node c. Since $\{a, b\}$ is a separation pair of $G, V_{0} \cap V_{1}^{\prime}=\{a, b\}$. But $c \in V_{0}$, thus $c \notin V_{1}^{\prime},\{a, c\} \cap V_{1}^{\prime}=\{a\}$, and $\{b, c\} \cap V_{1}^{\prime}=\{b\}$. Therefore $T$ is a tree decomposition of $G$.

Applying the same procedure for cases in rows three and four in Table 1 completes the proof.

\subsection{Domain equivalence}

Now, we will see that the class of s-tree decomposable geometric constraint graphs and the class of tree decomposable graphs are the same. In other words, a geometric constraint problem expressed by means of a geometric constraint graph is solvable by Owen's technique if and only if the graph is tree decomposable. Since we proved in [9] that a geometric constraint graph is solvable by reduction analysis, [5], if and only if the graph is tree decomposable, this implies that Owen's technique and reduction analysis have the same domain and that its domain can be characterized by the class of tree decomposable graphs.

Theorem 5.4 Let $G=(V, E)$ be a geometric constraint graph. The following assertions are equivalent:

1. G is tree decomposable.

2. $G$ is s-tree decomposable.
3. $G$ is solvable by reduction analysis.

4. G is solvable by decomposition analysis.

Proof

Joan-Arinyo et al. proved in [9] the equivalence of assertions 1, 3 and 4. Lemma 5.2 proves that 1 implies 2 and Lemma 5.3 proves that 2 implies 1.

\section{Summary}

We have introduce the concept of deficit of a constraint graph. Based on this concept, we have presented a new formalization for a decomposition analysis algorithm. We have proved its correctness. The idea of preserving just the constraint graph deficit avoids the need for general algorithms to compute triconnected components. Thus the resulting decomposition analysis algorithm is simpler both conceptually and from a computational point of view.

We have used the tree decomposition as a general tool for decomposition analysis of constraint graphs. Specifically, we have applied it to prove that different decomposition analysis formalizations solve the same class of geometric constraint problems.

\section{Acknowledgements}

This research has been supported by CICYT under the project TIC2001-2099-C03-01.

\section{References}

[1] Alfred V. Aho, John E. Hopcroft, and Jeffrey D. Ullman. The Design and Analysis of Computer Algorithms. Computer Science and Information Processing. Addison Wesley Publishing Company, Reading, MA, 1974.

[2] Gary Chartrand and Linda Lesniak. Graphs \& Digraphs. Chapman \& Hall, 3rd edition, 1996.

[3] C. Durand. Symbolic and Numerical Techniques for Constraint Solving. $\mathrm{PhD}$ thesis, Purdue University, Department of Computer Sciences, December 1998.

[4] I. Fudos. Editable representations for 2D geometric design. Master's thesis, Purdue University, Department of Computer Sciences, 1993.

[5] I. Fudos and C.M. Hoffmann. Correctness proof of a geometric constraint solver. Technical Report CSD 93-076, Department of Computer Sciences, Purdue University, December 1993.

[6] I. Fudos and C.M. Hoffmann. A graph-constructive approach to solving systems of geometric constraints. ACM Transactions on Graphics, 16(2):179-216, April 1997.

[7] C.M. Hoffmann, A. Lomonosov, and M. Sitharam. Geometric constraint decomposition. In B. Brüderlin and D. Roller, editors, Geometric Constraint Solving and Applications, pages 171-195. Springer, Berlin, 1998.

[8] J. E. Hopcroft and R. E. Tarjan. Dividing a graph into triconnected components. Technical report, Computer Science Department. Cornell University, Ithaca, NY. USA, February 1974. New revision of TR 72-140. 
[9] R. Joan-Arinyo, A. Soto-Riera, S. Vila-Marta, and J. Vilaplana. On the domain of constructive geometric constraint solving techniques. In R. Duricovič and S. Czanner, editors, Spring Conference on Computer Graphics, pages 49-54, Budmerice, Slovakia, April 25-28 2001. IEEE Computer Society, Los Alamintos, CA.

[10] G. Laman. On graphs and rigidity of plane skeletal structures. Journal of Engineering Mathematics, 4(4):331-340, October 1970.

[11] N. Mata. Solving incidence and tangency constraints in 2D. Technical Report LSI-97-3R, Department LSI, Universitat Politècnica de Catalunya, 1997.
[12] Gary L. Miller and Vijaya Ramachandran. A new graph triconnectivity algorithm and its parallelization. Combinatorica, 12:53-76, 1992.

[13] J.C. Owen. Algebraic solution for geometry from dimensional constraints. In ACM Symp Foundations of Solid Modeling, pages 397-407, Austin, TX, 1991.

[14] P. Todd. A k-tree generalization that characterizes consistency of dimensioned engineering drawings. SIAM J. Disc. Math, 2(2):255-261, 1989. 